\title{
Negative refractive index due to chirality
}

\author{
Jiangfeng Zhou, ${ }^{1}$ Jianfeng Dong, ${ }^{1,2}$ Bingnan Wang, ${ }^{1}$ Thomas \\ Koschny, ${ }^{1,3}$ Maria Kafesaki, ${ }^{3}$ and Costas M. Soukoulis ${ }^{1,3}$ \\ ${ }^{1}$ Ames Laboratory and Department of Physics and Astronomy, Iowa State University, Ames, Iowa 50011, USA \\ ${ }^{2}$ Institute of Optical Fiber Commun. and Network Tech., Ningbo University, Ningbo 315211, China \\ ${ }^{3}$ Institute of Electronic Structure and Laser - Foundation for Research and Technology Hellas (FORTH), \\ and Department of Materials Science and Technology, University of Crete, Greece
}

\begin{abstract}
We demonstrate experimentally and numerically that metamaterials based on bilayer cross wires give giant optical activity, circular dichroism, and negative refractive index. The presented chiral design offers a much simpler geometry and more efficient way to realize negative refractive index at any frequency. We also developed a retrieval procedure for chiral materials which works successfully for circularly polarized waves.
\end{abstract}

PACS numbers: $78.20 . \mathrm{Ek}, 41.20 . \mathrm{Jb}, 42.25 . \mathrm{Ja}$

Recently, chiral metamaterials are proposed as an alternative to realize negative refractive index. ${ }^{1,2,3}$ Chiral metamaterials are metamaterials made of unit cells without symmetry planes. It has been shown that backward

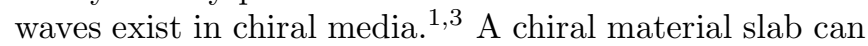
focus incident EM beams and can be used as a perfect lens. $\frac{4,5}{=}$ In 2004, the canonical helix ${ }^{3}$ and the twisted Swiss-role metal structures ${ }^{2}$ for microwave applications have been discussed as possible candidates to achieve negative refractive index. Later, the bilayer rosette-shaped chiral metamaterial is proposed and fabricated at mi-

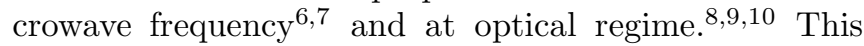
metamaterial exhibits a very strong rotary power in the microwave, near-infrared and visible spectral ranges. In the microwave spectral region, in terms of rotary power per wavelength, the bilayer structure rotates five orders of magnitude stronger than a gyrotropic crystal of quartz $z^{\underline{6}}$. It has been shown that the strong gyrotropy originates from the magnetic resonance of the bilayer metallic structure with anti-parallel current flowing in the bilayer metal wires ${ }^{11}$ In this sense, the bilayer chiral structure is the chiral version of the short wire pair ${ }^{12,13,14}$ type of metamaterials. The planar chiral structure $\frac{15,16}{16}$ and chiral photonic crystal 17 are proposed and fabricated. More recently, a chiral SRR consisting of double layers of SRRs is proposed to provide negative refractive index ${ }^{18}$.

In this letter we demonstrate, experimentally and numerically, the negative refraction using the bilayer crosswire design. We show the negative refraction originates from the $3 \mathrm{D}$ chiral properties of the bilayer cross wires. Unlike conventional negative index material designs, such as the split-ring resonator type design $\frac{19}{}$ and fishnet designs, 20 the chiral negative index material does not require simultaneously negative permittivity and permeability, and, therefore, the chiral design can offer much simpler geometry and a more efficient way to realize negative refraction index. Due to the asymmetric geometry of the cross-wire pairs, the cross coupling between the magnetic field and electric field happens at the chiral resonances and provides strong chirality around the resonance frequencies. Further study shows chiral res- onances are either electric resonance or magnetic resonance of short wire pairs. The negative refractive index of chiral metamaterial arises from this strong chirality, which splits the refractive indices, $n_{ \pm}$, of the two circularly polarized waves and makes the refractive index of one circular polarization become negative $\stackrel{2}{=}$ Our study shows that two resonance modes exist for the cross-wire pairs. The resonance mode at the lower frequency is a magnetic resonance mode with anti-parallel currents, while the resonance mode at the higher frequency is an electric resonance with parallel currents.
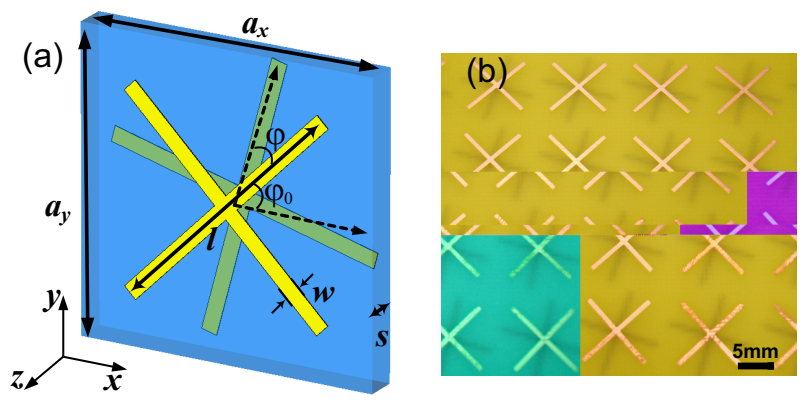

FIG. 1: (Color online) (a) Schematic representation of one unit cell of the cross-wire structure. (b) Photograph of one side of a fabricated microwave-scale cross-wire sample. The geometry parameters are given by $a_{x}=a_{y}=15 \mathrm{~mm}, l=$ $14 \mathrm{~mm}, w=0.8 \mathrm{~mm}, s=1.6 \mathrm{~mm}, \phi_{0}=45^{\circ}$, and $\phi=30^{\circ}$. The thickness of the copper is $t_{m}=36 \mu \mathrm{m}$.

We develop a retrieval procedure adopting the uniaxial bi-anisotropic model to calculate the effective parameters, $\mu, \epsilon, \kappa$ and $n_{ \pm}$, of the chiral metamaterial design. We prove the existence of the negative index originating from the chirality, $\kappa$ of the cross-wire metamaterial. As a comparison, the non-chiral version of the cross-wires pair design does not show any negative refractive index. Finally, we study the current distribution for both the electric and the magnetic resonances, and determine electric and magnetic resonances are the symmetric and antisymmetric resonance modes of the coupled short wire resonances, respectively. 
The layout of the proposed structure is shown in Fig. 1. An $18 \times 14$ array of cross wires is patterned on a double side copper-clad FR-4 board. The overall size of our sample is $279 \times 216 \mathrm{~mm}^{2}$. The dielectric constant of the FR-4 board is $\epsilon_{r}=4.5+0.15 \mathrm{i}$. The the properties of the crosswires shown in Fig. 1 are characterized, using simulations and microwave measurements, and these results are used to determine the expected properties of metamaterials built from the cross-wire building blocks. Simulations of cross-wire structures are achieved with CST Microwave Studio (Computer Simulation Technology GmbH, Darmstadt, Germany), which uses a finite element method to determine reflection and transmission properties. In the simulations, the periodic boundary conditions are applied to a single unit cell shown in Fig. 1(a). Since the eigen solutions of the electromagnetic (EM) wave in chiral materials are two circularly polarized EM waves, 21,22 i.e., the right-handed circularly polarized wave $(\mathrm{RCP},+)$ and the left-handed circularly polarized wave (LCP,-), correspondingly, four transmission coefficients, $\stackrel{23}{,} T_{++}, T_{-+}$, $T_{+-}$, and $T_{--}$, are obtained to fully characterize the response of the chiral metamaterials. In the experiment, the transmission coefficient is measured by HP $8364 \mathrm{~B}$ network analyzer with two Narda standard horn antennas. Four linear transmission coefficients, $T_{x x}, T_{y x}, T_{x y}$, and $T_{y y}$, are measured and the circular transmission coefficients, $T_{++}, T_{-+}, T_{+-}$, and $T_{--}$are converted from the linear transmission coefficients using the following equation,

$$
\begin{aligned}
& \left(\begin{array}{ll}
T_{++} & T_{+-} \\
T_{-+} & T_{--}
\end{array}\right)=\frac{1}{2} \times \\
& \left(\begin{array}{cc}
\left(T_{x x}+T_{y y}\right)+\mathrm{i}\left(T_{x y}-T_{y x}\right) & \left(T_{x x}-T_{y y}\right)-\mathrm{i}\left(T_{x y}+T_{y x}\right) \\
\left(T_{x x}-T_{y y}\right)+\mathrm{i}\left(T_{x y}+T_{y x}\right) & \left(T_{x x}+T_{y y}\right)-\mathrm{i}\left(T_{x y}-T_{y x}\right)
\end{array}\right)
\end{aligned}
$$

Figures 2(a) and (b) show the simulated and measured transmission coefficients, $T_{++}$and $T_{--}$, as a function of frequency, respectively (the cross coupling transmission, $T_{-+}$and $T_{+-}$, are negligible, not shown here). Due to the asymmetric geometry along the propagating direction, the transmission responses for RCP and LCP split into two curves. Notice two resonance dips are observed at frequencies, $f=6.5$ and $7.5 \mathrm{GHz}$, in both $T_{++}$and $T_{--}$curves. For the first resonance at $6.5 \mathrm{GHz}$, the transmission dip for RCP, $T_{++}$, is much deeper than that for LCP, $T_{--}$, which implies the resonance for RCP is much stronger than LCP. While, for the second resonance at 7.5 $\mathrm{GHz}$, we observe the resonance for LCP is much stronger than RCP. Use the standard definitions ${ }^{24}$ of the polarization azimuth rotation, $\theta=\left[\arg \left(T_{++}\right)-\arg \left(T_{--}\right)\right] / 2$, and the ellipticity, $\eta=\frac{1}{2} \arcsin \left(\frac{\left|T_{++}\right|-\left|T_{--}\right|}{\left|T_{++}\right|+\left|T_{--}\right|}\right)$, of elliptically polarized light, we calculate the polarization changes of a linearly polarized wave incident on the cross wire structures. The simulated and measured azimuth rotation, $\theta$, and ellipticity, $\eta$, are presented in Fig. 2(c),(e) and (d),(f), respectively. At the resonance frequencies of 6.5 and $7.5 \mathrm{GHz}$, the azimuth rotation and ellipticity reach their maximum values, $\left(\theta=-89^{\circ}, \eta=-28^{\circ}\right)$ and $\left(\theta=-130^{\circ}, \eta=28^{\circ}\right)$, respectively. In the region between two resonance dips (around $6.9 \mathrm{GHz}$ ), the region with low loss and nearly zero dichroism, we observes a polarization rotation of $-40^{\circ}$ with $\eta \approx 0$, about four times larger than the value reported using similar bi-layer chiral metamaterial designs, such as bilayer of twisted rosettes. 6.7 The sign change of $\eta$ at $7.0 \mathrm{GHz}$ reflects the different frequency dependence between the magnitude of the transmission $\left|T_{++}\right|$and $\left|T_{--}\right|$. As a consequence, the outgoing wave of a linear polarized incident wave below and above 7.0 GHz have different handedness. Our numerical simulations show that the differences between $\left|T_{++}\right|$and $\left|T_{--}\right|$result from the different amounts of loss as the RCP and LCP waves pass through the cross wire structures.

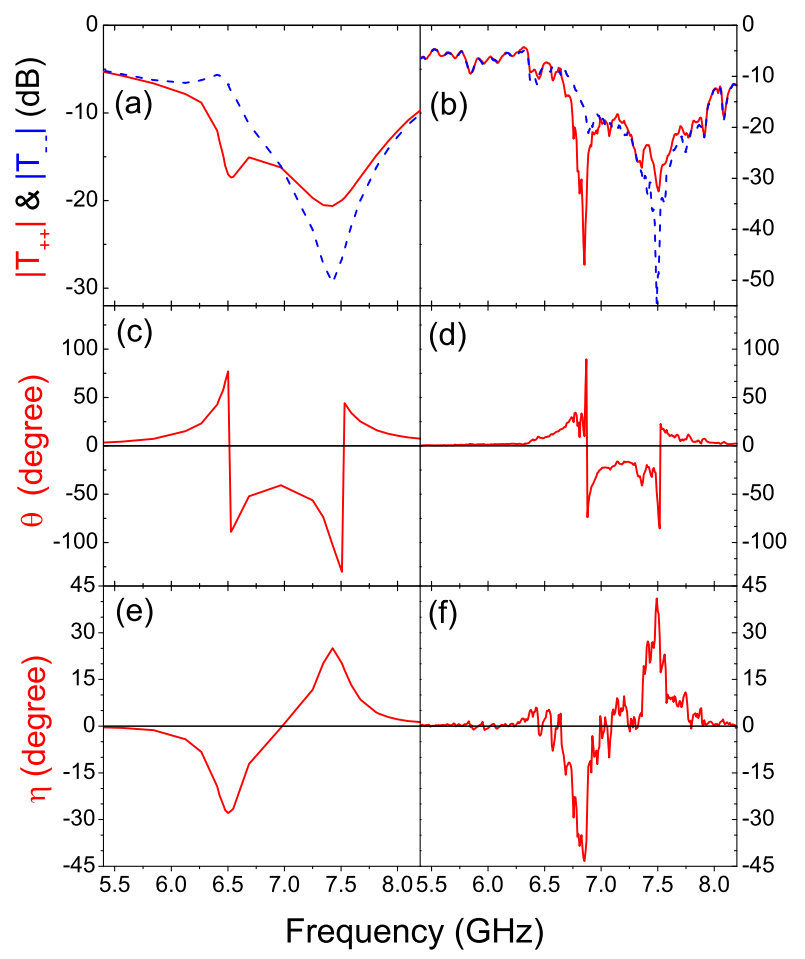

FIG. 2: (Color online) (a) and (b): Simulated and measured magnitude of the transmission coefficients for the right circularly polarized, $\left|T_{++}\right|$(red solid), and the left circularly polarized, $\left|T_{--}\right|$(blue dashed), electromagnetic wave, respectively. (c) and (d): Simulated and measured polarization azimuth rotation, $\theta$, respectively. (e) and (f): Simulated and measured ellipticity angle, $\eta$, respectively.

To study the effective parameters of chiral metamaterials, we develop a retrieval procedure based on a uniaxial bi-anisotropic model $\stackrel{7}{-}$ The cross wire pairs design can be modeled as a reciprocal uniaxial bi-anisotropic medium and the constitutive equation is given by

$$
\left(\begin{array}{l}
\mathbf{D} \\
\mathbf{B}
\end{array}\right)=\left(\begin{array}{cc}
\epsilon_{0} \epsilon & -\mathrm{i} \kappa / c_{0} \\
\mathrm{i} \kappa / c_{0} & \mu_{0} \mu
\end{array}\right)\left(\begin{array}{l}
\mathbf{E} \\
\mathbf{H}
\end{array}\right)
$$

where $\epsilon_{0}, \mu_{0}$, and $c_{0}$ are the permittivity, permeability, and the speed of light in a vacuum, respectively. Assuming $\exp (-i \omega t)$ time dependent, the eigen solution of the electromagnetic wave in bi-isotropic media is circular polarized plane waves, and the polarization is either 
left-handed circular polarized (LCP) or right-handed polarized (RCP). The refractive indices for LCP and RCP are given by 21,22

$$
n_{ \pm}=\sqrt{\epsilon \mu} \pm \kappa
$$

where $(+)$ and (-) denote RCP and LCP, respectively. Both LCP and RCP have the same impedance given by $z / z_{0}=\sqrt{\mu / \epsilon}$, where $z_{0}$ is the impendence of the vacuum.

From Eq. (3), one can immediately see that $n<0$ for one of the polarizations, if $\kappa$ is large enough, such that $\sqrt{\epsilon \mu}<\kappa$. It has been shown that the polarization azimuth rotation, $\theta$, is proportional to the chiral parameter, $\kappa$. Specifically, $\theta=\kappa k_{0} d$, where $k_{0}$ and $d$ are the wave vectors in a vacuum and the thickness of the bi-isotropic slab. Thus, the chiral materials with large rotary power, such as the bilayer structures and chiral SRRs, possibly possess the negative refractive index. However, it is not trivial to obtain negative refractive index by a chiral design with a large azimuth rotation. One should keep in mind that the large value of azimuth angle, $\theta$, happens at the resonances, where $\sqrt{\epsilon \mu}$ is also large, so $\kappa$ should be large enough to overcome the large magnitude of $\sqrt{\epsilon \mu}$ to achieve negative $n$.

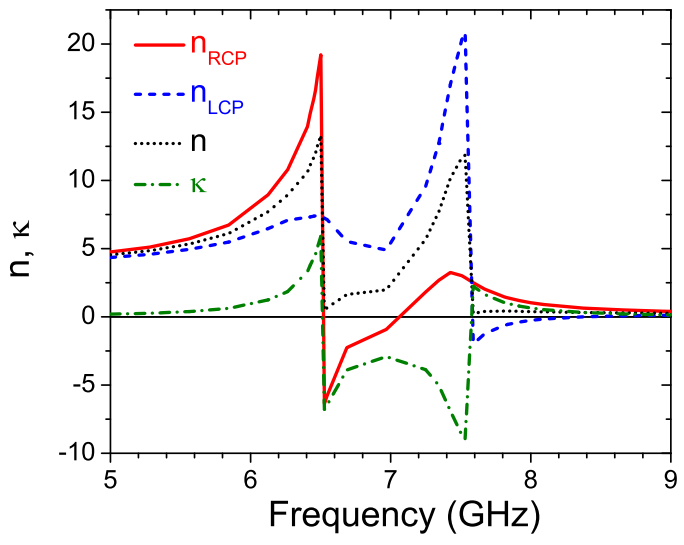

FIG. 3: (Color online) The retrieved effective refractive index for the right circularly polarized EM wave, $n_{+}$(red solid), and the left circularly polarized EM wave, $n_{-}$(blue dashed). The black dotted curve shows the refractive index calculated by the permittivity and the permeability, $n=\sqrt{\epsilon \mu}$, and the green dash-dotted curve shows the chiral parameter, $\kappa$.

In Fig. 3, we present the refractive index for RCP and LCP, $n_{+}, n_{-}$, the conventional definition of refraction index, $n=\sqrt{\epsilon \mu}$, and the chiral parameter, $\kappa$, which are extracted from simulation results using the retrieval procedure. A thickness, $d=s+2 t_{m}=1.672 \mathrm{~mm}$, is used when we extract these effective parameters. Notice that $n$ (black dotted curve) is positive through the entire frequency range from 5 to $9 \mathrm{GHz}$. However, $n_{+}$(red solid) is negative from 6.5 to $7.0 \mathrm{GHz}$ and $n_{-}$(blue dashed) has a negative region from 7.6 to $8.2 \mathrm{GHz}$. The chiral parameter (green dash-dotted curve) shows two resonances at 6.5 and $7.5 \mathrm{GHz}$, respectively. Above the first resonance frequency, $\kappa$ is negative between 6.5 to $7.0 \mathrm{GHz}$, which leads to $n_{+}=\sqrt{\epsilon \mu}+\kappa<0$ between 6.5 to $7.0 \mathrm{GHz}$ . Similarly, above the second resonance frequency, $\kappa$ is positive and results in $n_{-}=\sqrt{\epsilon \mu}-\kappa<0$ between 7.6 to 8.2 GHz. It is clear the negative refractive index for RCP and LCP originates from the chiral parameter, $\kappa$. The observed negative refractive index $n_{+}=-2.5$ at $6.8 \mathrm{GHz}$ has the figure of merit, $\mathrm{FOM}=|\operatorname{Re}(n) / \operatorname{Im}(n)|=0.75$, and $n_{-}=-1$ at $7.8 \mathrm{GHz}$ has $\mathrm{FOM}=0.5$. The figure of merit is relatively low compared to the conventional negative index material designs such as SRRs or fishnet. The low FOM is due to the high loss of this chiral metamaterial design. Further study shows the loss mainly originates from the lossy dielectric spacer. If low loss dielectric materials is used in the cross wire design, the FOM can improve substantially. For instance, in our numerical simulations, we obtained $\mathrm{FOM} \approx 10$ using dielectric spacer with $\epsilon_{r}=4.5+0.005 i$.

We also calculate the $n_{+}, n_{-}, n$ and $\kappa$ for a non-chiral cross-wire pairs with the mutual twisted angle $\phi=0$. The results show $n_{+}=n_{-}=n>0$, and the chiral parameters, $\kappa=0$ through the entire frequency range from 5 to $9.0 \mathrm{GHz}$. Thus, we confirme the negative refractive index observed in Fig. 3 is due to the chirality introduced by the asymmetry of the cross-wire pairs.

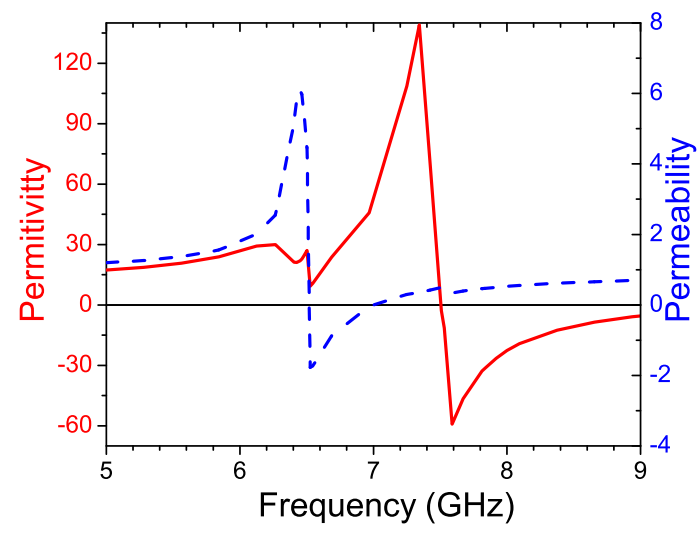

FIG. 4: (Color online) The real part of the effective permittivity (red solid) and the effective permeability (blue dashed), extracted from the simulation data.

In Fig. 4 we present the real parts of the permittivity, $\epsilon$, and the permeability, $\mu$, as a function of frequency for the asymmetric cross wire pairs. Two resonances are observed in $\mu$ (blue dashed) at 6.5 and $\epsilon$ (red solid) at $7.5 \mathrm{GHz}$, respectively. This demonstrates the resonance observed in Figs. 2 and 3 at $6.5 \mathrm{GHz}$ is a magnetic resonance and the resonance at $7.5 \mathrm{GHz}$ is an electric resonance. The magnetic resonance gives the negative $\mu$ between 6.5 to $6.9 \mathrm{GHz}$, and the electric resonance give a negative $\epsilon$ between 7.6 to $9 \mathrm{GHz}$. There is no overlap region of negative $\epsilon$ and $\mu$. Therefore, $\operatorname{Re}(n)=\operatorname{Re}(\sqrt{\epsilon \mu})>0$, which is consistent with the observation of $\operatorname{Re}(n)>0$ shown in Fig. 3 .

In order to understand the mechanism of the resonances for the cross wire pair design, we studied the 

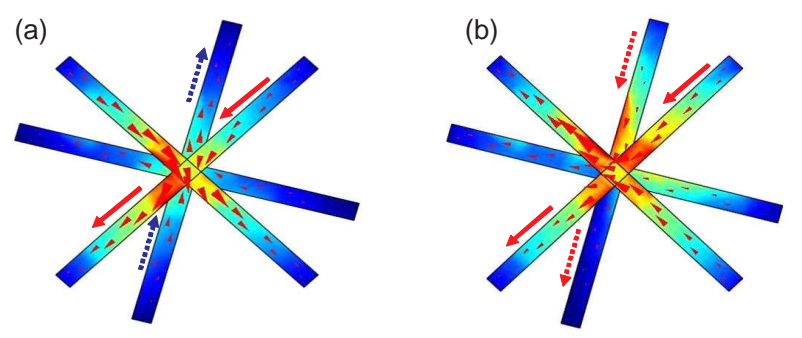

FIG. 5: (Color online) The simulated current density distribution for the right circularly polarized EM wave at $6.5 \mathrm{GHz}$ (a) and for the left circularly polarized EM wave at $7.5 \mathrm{GHz}$ (b). The cones (in red) show the direction and magnitude of the current density. The magnitude of the current density is also shown by the color on the wires, with red and blue corresponding to largest and smallest values.

current density distribution as shown in Fig. 5. Notice, at the magnetic resonance, the anti-parallel current exists on the top and bottom layer of cross-wire pairs (Fig. 5(a)), which is an asymmetric resonance modes. In Fig. 5(b), one can see parallel currents flowing on the two layers of wires, which is a symmetric resonance mode. The current distribution shows that the cross wire pairs can be viewed as a chiral version of the short wire pairs, $, 12,13,14$ which has similar current distributions in the symmetric and asymmetric resonance modes.

In summary, we experimentally demonstrated bilayer cross wires with very adaptable properties, including negative index of refraction due to chirality, giant optical activity and very large circular dichroism. We performed numerical simulations that give evidence that the negative refractive index is due to the chiral nature of the metamaterial and not from the simultaneous negative $\epsilon$ and $\mu$ as for conventional negative index materials. In addition, we developed a retrieval procedure that works well for chiral metamaterials. The geometry of the cross wire design is simple and easy-to-fabricate, and therefore, is more suitable for optical frequency applications compared to other types of bi-layer chiral metamaterial designs.

Work at Ames Laboratory was supported by the Department of Energy (Basic Energy Sciences) under contract No. DE-AC02-07CH11358. This work was partially supported by the Department of Navy, Office of the Naval Research (Award No. N00014-07-1-0359), European Community FET project PHOME (Contract No. 213390) and AFOSR under MURI grant (FA 9550-06-1-0337). The author Jianfeng Dong gratefully acknowledges support of the W.C. Wong Education Foundation, Hong Kong, the National Basic Research Program (973) of China (Grant No. 2004CB719805) and the National Natural Science Foundation of China (Grant No.60777037).
1 S. Tretyakov, I. Nefedov, A. Sihvola, S. Maslovski, and C. Simovski, J. Electromag. Waves Appl. 17, 695 (2003).

2 J. B. Pendry, Science 306, 1353 (2004).

3 S. Tretyakov, A. Sitivola, and L. Jylha, Photon. Nanostructures-fundamentals Applications 3, 107 (2005).

${ }^{4}$ Y. Jin and S. He, Opt. Express 13, 4974 (2005).

${ }^{5}$ C. Monzon and D. W. Forester, Phys. Rev. Lett. 95, 123904 (2005).

6 A. V. Rogacheva, V. A. Fedotov, A. S. Schwanecke, and N. I. Zheludev, Phys. Rev. Lett. 97, 177401 (2006).

7 E. Plum, J. Zhou, J. Dong, V. A. Fedotov, T. Koschny, C. M. Soukoulis, and N. I. Zheludev, Phys. Rev. B 79, 035407 (2009).

8 E. Plum, V. A. Fedotov, A. S. Schwanecke, N. I. Zheludev, and Y. Chen, Appl. Phys. Lett. 90, 223113 (2007).

9 T. Vallius, K. Jefimovs, J. Turunen, P. Vahimaa, and Y. Svirko, Appl. Phys. Lett. 83, 234 (2003).

10 M. Decker, M. W. Klein, M. Wegener, and S. Linden, Opt. Lett. 32, 856 (2007).

11 Y. Svirko, N. Zheludev, and M. Osipov, Appl. Phys. Lett. 78, 498 (2001).

12 V. M. Shalaev, W. S. Cai, U. K. Chettiar, H. K. Yuan, A. K. Sarychev, V. P. Drachev, and A. V. Kildishev, Opt. Lett. 30, 3356 (2005).

13 J. Zhou, L. Zhang, G. Tuttle, T. Koschny, and C. M. Soukoulis, Phys. Rev. B 73, 041101(R) (2006).
14 G. Dolling, C. Enkrich, M. Wegener, J. F. Zhou, and C. M. Soukoulis, Opt. Lett. 30, 3198 (2005).

15 V. A. Fedotov, P. L. Mladyonov, S. L. Prosvirnin, A. V. Rogacheva, Y. Chen, and N. I. Zheludev, Phys. Rev. Lett. 97, 167401 (2006).

16 B. Bai, Y. Svirko, J. Turunen, and T. Vallius, Phys. Rev. A 76, 023811 (2007).

17 M. Thiel, G. von Freymann, and M. Wegener, Opt. Lett. 32, 2547 (2007).

18 L. Jelinek, R. Marqués, F. Mesa, and J. D. Baena, Phys. Rev. B 77, 205110 (2008).

19 D. Smith, W. Padilla, D. Vier, S. Nemat-Nasser, and S. Schultz, Phys. Rev. Lett. 84, 4184 (2000).

20 G. Dolling, C. Enkrich, M. Wegener, C. M. Soukoulis, and S. Linden, Science 312, 892 (2006).

21 J. A. Kong, Electromagnetic Wave Theory (Cambridge, 2000).

22 C. Monzon, IEEE Trans. Antennas Propag. 38, 227 (1990).

23 In these notations, the first and second subscripts indicate the output and input signal polarization, respectively. For example, $T_{+-}=E_{+}^{t} / E_{-}^{i}$, where $E_{-}^{i}$ is the input LCP electric field and $E_{+}^{t}$ is the transmitted RCP electric field.

24 J. D. Jackson, Classical Electrodynamics (John Wiley, 1998), 3rd ed. 